\title{
Treating Acne in Obese and Morbidly Obese Patients With Tazarotene 0.045\% Lotion: Post Hoc Analysis of Pooled Phase 3 Data
}

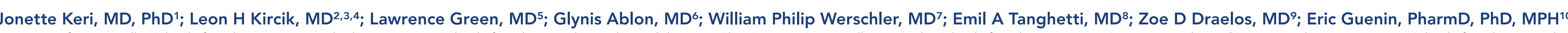

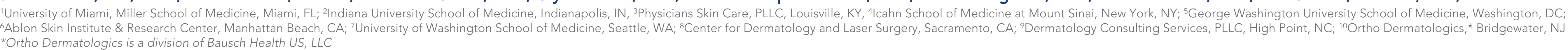

\section{SYNOPSIS}

- Acne prevalence may be higher in obese individuals, potentially due to hormonal, inflammatory, and/or dietary factors. ${ }^{1,2}$

However, the effect of obesity on the efficacy and safety of topical acne treatments is largely unknown The growing population of obese individuals in the United States, along with the negative impact of high body mass index (BMI) and acne on health-related quality of life, underscores the need to identify

A new lower-dose tazarotene 0.045\% lotion

A new, lower-dose tazarotene $0.045 \%$ lotion

developed utilizing polymeric emulsion technology

- This easily spreadable lotion formulation allows for This easily spreadable lotion formulation allows for
more efficient delivery of tazarotene into dermal layers while reducing potential skin irritation

OBJECTIVE

To evaluate efficacy, safety, and impact on quality of Ife of tazarotene $0.045 \%$ lotion in obese and morbidly obese individuals with acne

METHODS

In two identical phase 3 randomized, double-blin vehicle-controlled studies (NCT03168321 and NCT3168334), participants aged $\geq 9$ years with
moderate-to-severe acne (Evaluator's Global Severity Score [EGSS] of 3 or 4 ) were randomized (1:1) to oncedaily tazarotene $0.045 \%$ lotion or vehicle lotion for 12 weeks

- In these studies, CeraVe ${ }^{\circledast}$ hydrating cleanser and CeraVe ${ }^{\circledR}$ moisturizing lotion (L'Oreal, NY) were provided as needed for optimal moisturization cleaning of the skin

- Outcomes comprised reductions in inflammatory and noninflammatory lesion counts, treatment success

(proportion of participants achieving $\geq 2$-grade

or 1 [almost clear]), and Acne-Specific Quality of Life (Acne-QoL) questionnaire

- Cutaneous safety and tolerability were also evaluated

Pooled data at week 12 were analyzed for the BMI
RESULTS

Participants

A total of 332 participants with $\mathrm{BMI} \geq 30 \mathrm{~kg} / \mathrm{m}^{2}$ were included for analysis

- Of these, one-fifth $(n=70)$ were morbidly obese $\left(B M I \geq 40 \mathrm{~kg} / \mathrm{m}^{2}\right)$

- The $B M I \geq 30 \mathrm{~kg} / \mathrm{m}^{2}$ subgroup, compared to the overall pooled population ( $\left.\mathrm{N}=16144^{6}\right)$, had more participants who were female $(79.5 \%$ vs $65.9 \%)$, EGSS score of $3(93.1 \%$ vs $90.9 \%$

Efficacy

- Tazarotene-treated obese and morbidly obese participants had an approximately

$55 \%$ reduction in both inflammatory and noninflammatory lesions following 12 weeks of treatment (Figure 1)

Results were comparable to reductions seen in the overall population $(57.9 \%$ and $56.0 \%$ reduction, respectively

At week 12, just over one-third of participants treated with tazarotene $0.045 \%$ Ption achieved treatment success $\left(\mathrm{BMI} \geq 30 \mathrm{~kg} / \mathrm{m}^{2}\right.$ TAZ vs vehicle. $33.5 \%$ vs $21.9 \%$ $P<0.05)$, similar to the overall population $(30.4 \%$ vs $17.9 \%$; $P<0.001)$ FIGURE 1. Lesion Reductions in Participants With BMI $\geq 30 \mathrm{~kg} / \mathrm{m}^{2}$ (ITT Population, Pooled)

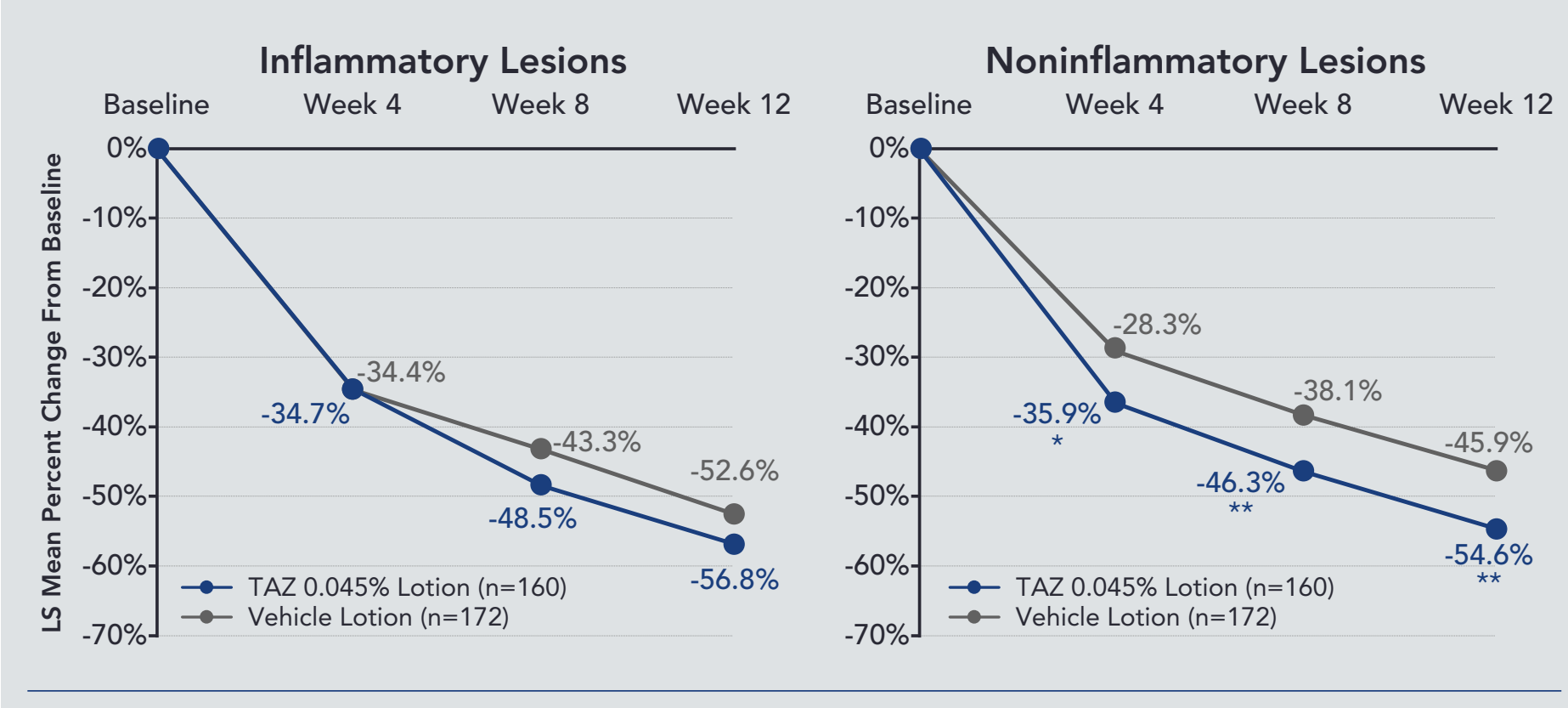

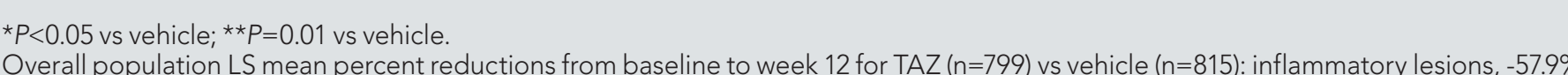

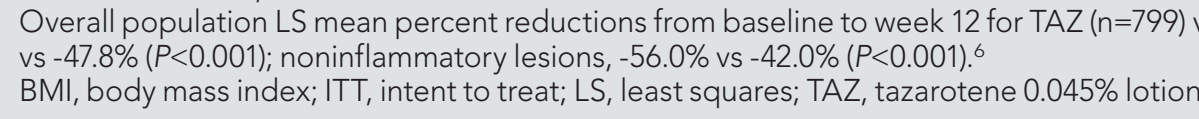

Quality of Life

Acne-QoL domain scores improved from baseline to week 12 in obese and Acne-OoL domains of "self-perception" and "acne symptoms" (Figure 2) - Improvements across all four Acne-OoL domains following tazarotene treatment population ${ }^{7}$

\section{Safety}

- Mean cutaneous safety and tolerability scores were low for both tazarotene

- Wit

With both tazarotene and vehicle, mean scores at baseline and week 12 were

Whe

- Slight improvements at week 12 versus baseline were observed for erythema,

- Resultswere simili to te overall population (data not shown)

FIGURE 2. Acne-QoL Improvements at Week 12 in Participants With

Acne-Ool Domains

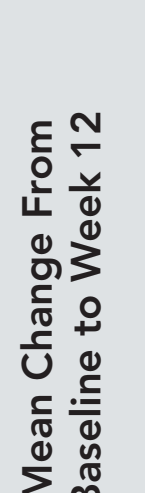

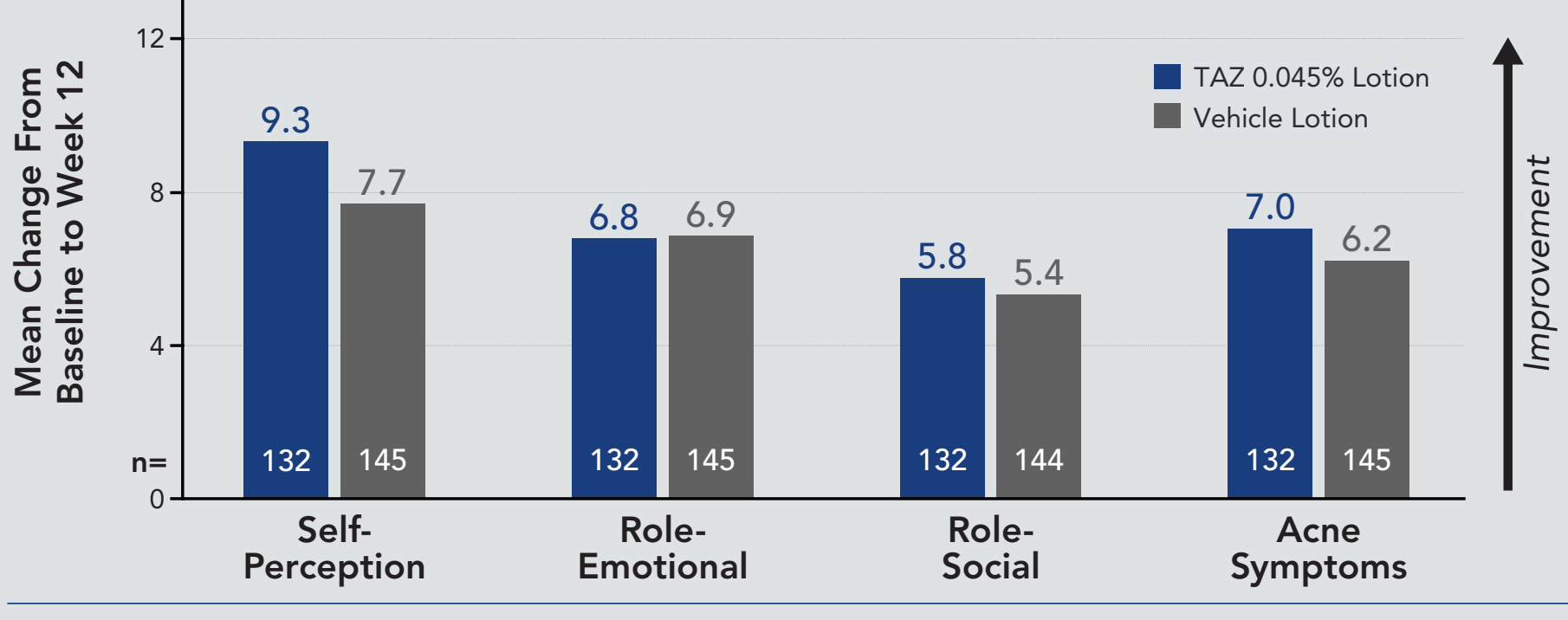

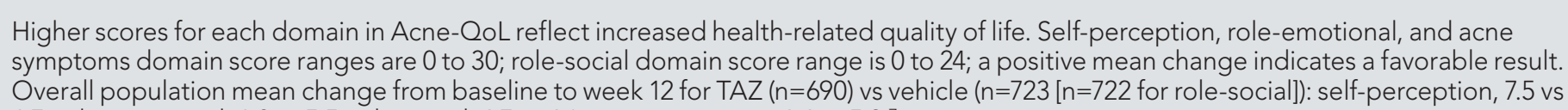

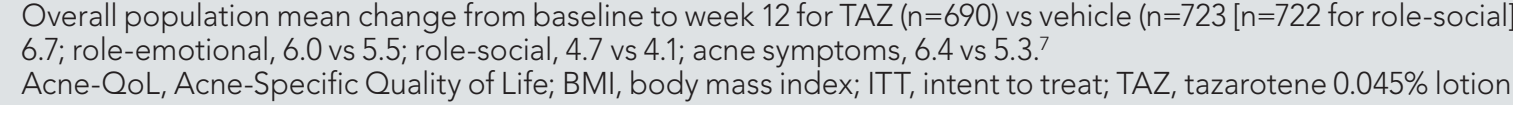

FIGURE 3. Mean Cutaneous Safety and Tolerability Scores in Participants With $\mathrm{BMI} \geq 30 \mathrm{~kg} / \mathrm{m}^{2}$ (Safety Population, Pooled)

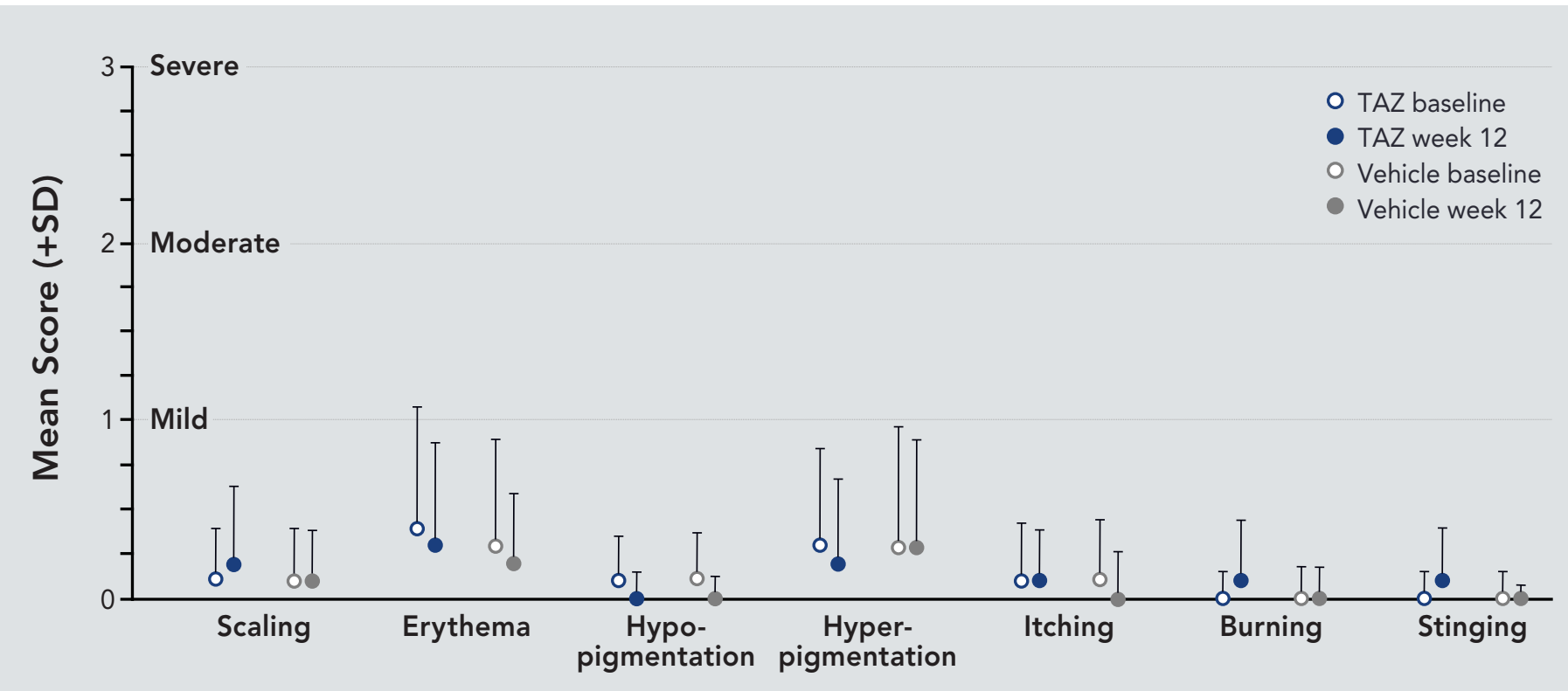

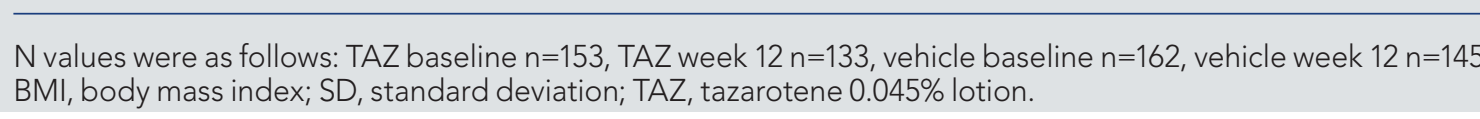

\section{CONCLUSIONS}

- In obese and morbidly obese participants with moderate-to-severe ache, tazarotene $0.045 \%$ lotion reduced inflammatory and noninflammatory lesions by over half, with one-third of participants achieving clear/

- Quality of life improvements with tazarotene $0.045 \%$ lotion were substantially greater in obese and morbidly obese participants versus
the overall study population

- These results may be due to both the worse higher percentage of females in the $\geq 30 \mathrm{~kg} / \mathrm{m}^{2}$ group compared with the overall population

- Studies have shown that females with acne are more likely to develop anxiety/ depression, and improvements in acne positively affect quality of life ${ }^{8}$. furthermore, females have been shown to have relatively greater quality of life improvements with tazarotene $0.045 \%$ lotion than males

- Cutaneous safety and tolerability scores with tazarotene lotion were generally simila

between baseline and week 12

- Tazarotene $0.045 \%$ lotion was efficacious and well tolerated in obese and morbidly obese individuals with acne, and led to large mprovements in the quality of life of these

- These results are notable given the limited clinical data of acne treatments in this
growing population

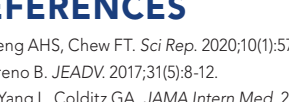

AUTHOR DISCLOSURES

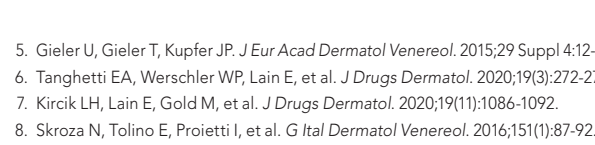

